\title{
NONUNIFORM CURRENTS FLOWING ON A PERFECTLY CONDUCTING CYLINDER
}

\author{
Hüsnü Deniz Bașdemir \\ Cankaya University, Engineering Faculty, Electronic and Communication Dept., Tel: +90 (312) 28445 00, Fax: +90 (312) 285 96 31, Balgat, Ankara, Turkey \\ basdemir@cankaya.edu.tr
}

\begin{abstract}
In this paper, we applied physical theory of diffraction (PTD) method to the perfectly electric conducting (PEC) cylinder for investigation of the scattering fields. Physical optics (PO) method was used to gain the uniform scattering field which is produced by the induced current from the incident wave on the tangential plane of the perfectly electric conducting cylinder. Nonuniform current and scattered field will be obtained by applying PTD method to the problem. As a last step of solution, nonuniform currents and nonuniform scattering fields were obtained with the assistance of the exact solution of the scattering waves from the cylinder. The scattering fields and currents are plotted numerically.
\end{abstract}

Keywords: Surface Diffraction, Fringe Currents

\section{Introduction}

Physical optics (PO) is a well known high frequency integral based technique that was suggested by Mcdonald in 1912 [1]. It is used for the investigation of the scattering from large metallic objects. It is suitable for acoustic and electromagnetic waves. In the acoustic this technique is known extended Kirchoff approximation. It can be accepted that the surface of the scattering object is an infinite tangential plane with respect to small wavelength according to obstacle. Main idea is to form an induced current on the scatterer. The current induced by the incident field is the source of the scattering fields. PO does not include diffraction phenomena due to the definition of $\mathrm{PO}$ on the lit region of the scatter surface. According to PO, the fields are equal to zero in the shadow region. Since the contribution of the nonuniform part is rejected, the technique is inadequate. PTD is used to fix the technique. PTD was suggested by Ufimtsev in 1950's [2]. While Ufimtsev was improving PO, he was aware of the Sommerfeld's exact wedge solution [3]. The fundamental idea of Ufimtsev's concept is to divide the induced current into two components. First component is obtained by using PO hence it is known as uniform current. Second component which is also known as nonuniform current is the source of the fields that radiate from discontinuities of the scatterer. This current occurring on the discontinuities is called fringe current and scattered field from discontinuities is called fringe field. These names were given by Ufimtsev. He obtained the nonuniform field by subtracting PO scattered field from the Sommerfeld's exact solution. To our knowledge there is no work in the literature about the surface diffraction using the method of PTD for the evaluation of the scattered fields from the cylinder. Using this method, nonuniform currents and nonuniform fields will be obtained and the contribution of these mentions to the scattering field and the impacts of the surface diffraction will be investigated. The time factor of $\exp (j \omega t)$ is assumed and suppressed throughout the paper, $\omega$ is the angular frequency.

\section{Theory}

The problem of the diffraction of planes waves from PEC cylinder is presented. The problem's geometry is given in the Fig. 1. The incident electric field is given by

$$
\vec{E}_{i}=\vec{e}_{z} E_{0} e^{-j k \rho \cos \phi}
$$

where $E_{0}$ is constant amplitude factor. 


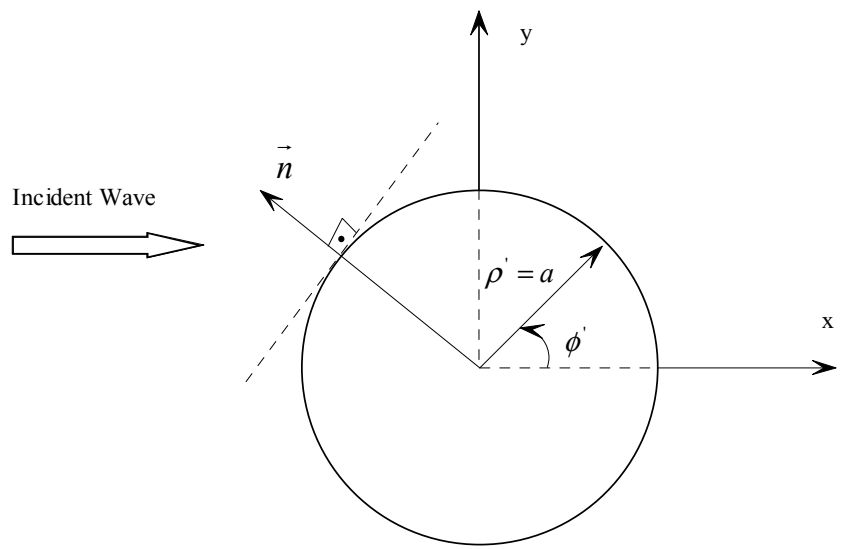

Fig. 1. The Geometry of the problem

PO current or uniform current can be written as

$$
\vec{J}_{P O}=2 \vec{n} \times\left.\vec{H}_{i}\right|_{s}
$$

where $\vec{n}$ is the normal vector and $\vec{H}_{i}$ is the magnetic field component of the incident field. The incident magnetic field is found to be

$$
\vec{H}_{i}=\frac{E_{0} k}{\omega \mu_{0}} e^{-j k \rho \cos \phi}\left(\vec{e}_{\rho} \sin \phi+\vec{e}_{\phi} \cos \phi\right)
$$

by using the Maxwell-Faraday equation. Under the surface condition, uniform current can be written as

$$
\vec{J}_{P O}=\vec{e}_{z} \frac{2 E_{0}}{Z_{0}} \cos \phi e^{-j k a \cos \phi}
$$

where $Z_{0}$ is equal to impedance of the vacuum. PO scattering surface integral is constructed as

$$
\vec{A}=\vec{e}_{z} \frac{E_{0} \mu_{0} a}{Z_{0} 2 \pi} \int_{z^{\prime}=-\infty}^{\infty} \int_{\phi^{\prime}=\frac{\pi}{2}}^{\frac{3 \pi}{2}} \cos \phi^{\prime} \frac{e^{-j k R}}{R} d \phi^{\prime} d z^{\prime}
$$

where $a$ is the radius of the cylinder. The $z$ ' part can be evaluated from Ref. [4] and it gives us Hankel function. Uniform scattering electric field can be written as

$$
\vec{E}_{s}^{P O} \cong \vec{e}_{z} \frac{-\omega \mu_{0} E_{0} a}{2 Z_{0}} \int_{\phi^{\prime}=\frac{\pi}{2}}^{\frac{3 \pi}{2}} \cos \phi^{\prime} e^{-j k a \cos \phi^{\prime}} H_{0}^{(2)}\left(k R_{1}\right) d \phi^{\prime} .
$$

According to PTD the total scattered field can be expressed as

$$
\vec{E}_{s}^{\text {total }}=\vec{E}_{s}^{P O}+\vec{E}_{s}^{N U}
$$

for $\vec{E}_{s}^{N U}$ is the nonuniform scattered field component. The total scattered electric field by the cylinder can be written from Ref. [5] as

$$
\vec{E}_{s}^{\text {total }}=-\vec{e}_{z} E_{0} \sum_{n=-\infty}^{\infty} j^{-n} \frac{J_{n}(k a)}{H_{n}^{(2)}(k a)} H_{n}^{(2)}(k \rho) e^{j n \phi}
$$


where $J_{n}$ is the zeroth order Bessel function and $H_{n}$ is called as the Hankel function. The nonuniform electric field can be written as

$$
\vec{E}_{s}^{N U}=\vec{E}_{s}^{\text {total }}-\vec{E}_{s}^{P O}
$$

according to Eq. (7) . Nonuniform scattered electric field can be written as

$$
\vec{E}_{s}^{N U}=-\vec{e}_{z} E_{0}\left\{\sum_{n=-\infty}^{\infty} j^{-n} \frac{J_{n}(k a)}{H_{n}^{(2)}(k a)} H_{n}^{(2)}(k \rho) e^{-j n \phi}-\vec{e}_{z} \frac{k a E_{0}}{2} \int_{\phi=\frac{\pi}{2}}^{\frac{3 \pi}{2}} \cos \phi^{\prime} e^{-j k a \cos \phi^{\prime}} H_{n}^{(2)}\left(k R_{1}\right) d \phi^{\prime}\right\}
$$

where $\omega \mu_{0}=k Z_{0}$ is taken into account. As the same procedure nonuniform surface currents is written as

$$
\vec{J}_{N U}=\vec{J}_{\text {exact }}^{\text {surface }}-\vec{J}_{P O}
$$

according to the PTD. Exact surface current can be written as

$$
\vec{J}_{\text {exact }}^{\text {surface }}=-\vec{e}_{z} \frac{2 E_{0}}{\omega \mu_{0} \pi a} \sum_{n=-\infty}^{\infty} \frac{j^{-n} e^{j n \phi}}{H_{n}^{(2)}(k a)}
$$

according to Ref. [5]. Nonuniform surface current takes the form

$$
\vec{J}_{N U}=-\vec{e}_{z} \frac{2 E_{0}}{\pi a k Z_{0}} \sum_{n=-\infty}^{\infty} \frac{j^{-n} e^{j n \phi}}{H_{n}^{(2)}(k a)}-\vec{e}_{z} \frac{2 E_{0}}{Z_{0}} \cos \phi e^{-j k a \cos \phi}
$$

when Eq. (12) is used.

\section{Numerical Results}

In this analysis part we will investigate the nonuniform current and scattered field. The distance between the observation point and origin will be taken as $6 \lambda$ which is constant for all plots and $\lambda$ is the wavelength. Radius of the cylinder $(a)$ will be taken as $2 \lambda$ for all plots. Figure 2 shows the currents on the cylinder. The amplitudes of exact and uniform currents are between $120^{\circ}$ and $240^{\circ}$. Maximum amplitude is obtained at $180^{\circ}$. Nonuniform current is the difference of the total current and PO current. As it can be seen from the Figure 2, the amplitude differences occur on the region bordered by brown line. Deficient current amplitude between the PO current and the exact current is accomplished by the nonuniform current amplitude. Figure 3 shows the scattered fields. Nonuniform scattered field takes the values at the first and fourth regions. PO radiation exists only at lit region which is bounded by angles $90^{\circ}$ and $270^{\circ}$ and the scattered fields from the shadow part of the obstacle are ignored. The exact total scattered electric field, occurring in all directions, from the PEC cylinder is shown in the Figure 3. The absent part of the exact field is accomplished by the nonuniform scattered field. 


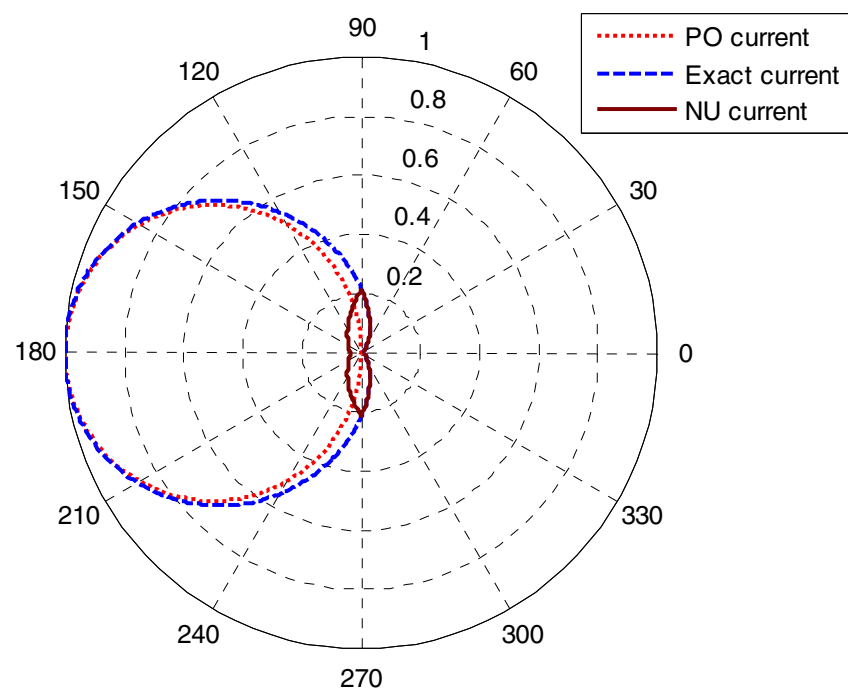

Fig. 2. Currents

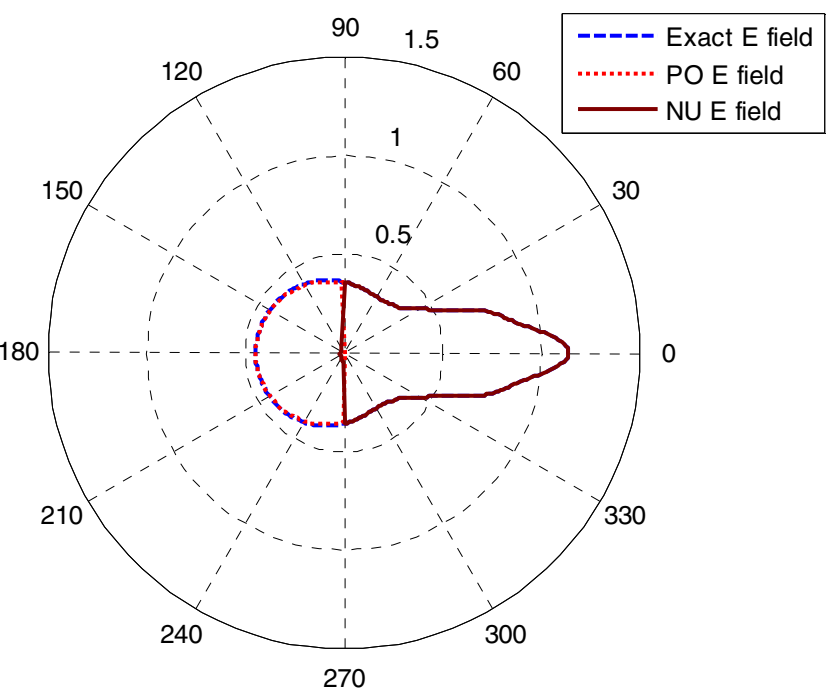

Fig. 3. Scattered Electric Field

\section{Conclusion}

In this study, the nonuniform currents flowing on the PEC cylinder and the nonuniform scattered electric field from this current were investigated by the method of PTD. Surface diffraction and the contribution of nonuniform parts to the scattering were observed from the numerical plots. Consistency of the results is compared with exact solutions; it is observed that the solutions are consistent with the exact solutions. It is seen that the contribution of the nonuniform parts are not negligible for compensation of the scattered field to the shadow regions.

\section{References}

1. H. M. Macdonald, "The Effect Produced by an Obstacle on a Train of Electric Waves," Phil. Trans. Royal Soc. London, Series A, Math. Phys. Sc., 212, 1913, pp. 299-337.

2. P. Ya. Ufimtsev, "Fundamentals of the Physical Theory of Diffraction,” New Jersey, Wiley.

3. A. Sommerfeld, "Mathematische Theorie der Diffraction," Math. Ann. 47, 1896, pp. 317-374.

4. Y. Z. Umul, "Modified Theory of Physical Optics," Opt. Express, vol. 12, no.20, September 2004, pp. 49594972 .

5. R. F. Harrington, "Time-Harmonic Electromagnetic Fields," IEEE Press Series on Electromagnetic Wave Theory, New York, Wiley, 2001. 